\title{
Protein expression of programmed death I ligand $I$ and ligand 2 independently predict poor prognosis in surgically resected lung adenocarcinoma
}

\author{
This article was published in the following Dove Press journal: \\ OncoTargets and Therapy \\ 12 April 2014 \\ Number of times this article has been viewed
}

\author{
Yang Zhang 1,2,* $^{2}$ \\ Lei Wang ${ }^{1,2, *}$ \\ Yuan $\mathrm{Li}^{2,3}$ \\ Yunjian $\operatorname{Pan}^{1,2}$ \\ Rui Wang ${ }^{1,2}$ \\ Haichuan $\mathrm{Hu}^{1,2}$ \\ Hang $\mathrm{Li}^{1,2}$ \\ Xiaoyang Luo ${ }^{1,2}$ \\ Ting Ye ${ }^{1,2}$ \\ Yihua Sun ${ }^{1,2}$ \\ Haiquan Chen ${ }^{1,2}$ \\ 'Department of Thoracic Surgery, Fudan \\ University Shanghai Cancer Center, \\ Shanghai, People's Republic of China; \\ ${ }^{2}$ Department of Oncology, Shanghai \\ Medical College, Fudan University, \\ Shanghai, People's Republic of China; \\ ${ }^{3}$ Department of Pathology, Fudan \\ University Shanghai Cancer Center, \\ Shanghai, People's Republic of China \\ *These authors contributed equally \\ to this work
}

Correspondence: Haiquan Chen Department of Thoracic Surgery, Fudan University Shanghai Cancer Center, 270 Dong-An Road, Shanghai 200032,

People's Republic of China

Tel +86 2I 64I75590

Fax +86216268 651I

Email hqchen I@yahoo.com

Yihua Sun

Department of Thoracic Surgery, Fudan University Shanghai Cancer Center,

270 Dong-An Road, Shanghai 200032,

People's Republic of China

Tel +86 2I 64175590

Fax +86 2I $626865 \mathrm{II}$

Email sun_yihua76@hotmail.com
Background: The clinicopathologic characteristics of tumors expressing programmed death (PD-1) ligands (PD-Ls) PD-L1 or PD-L2 and their associations with common driver mutations in lung adenocarcinoma are not clearly defined, despite the progression of anti-PD-1/PD-L1 immunotherapy.

Methods: PD-L1 and PD-L2 expression was measured by immunohistochemistry in 143 surgically resected lung adenocarcinomas and was correlated with clinical variables, histologic subtypes, and the mutational status of EGFR, KRAS, HER2, and ALK.

Results: Positive PD-L1 expression was significantly associated with more advanced T status, $\mathrm{N}$ status, and pathologic stage. Histologically, lung adenocarcinomas with positive PD-L1 staining were less likely to be adenocarcinoma in situ or minimally invasive adenocarcinoma and more likely to have solid predominant subtype. Both PD-L1 expression (odds ratio $=1.984$, 95\% confidence interval $=1.010-3.894 ; P=0.047$ ) and PD-L2 expression (odds ratio $=2.328$, $95 \%$ confidence interval $=1.201-4.512 ; P=0.012$ ) were independent predictors of poor overall survival. When the combined PD-L expression and pathologic stage were used together to predict overall survival, the concordance index increased to 0.763 , and the Akaike information criteria value decreased to 356.08 .

Conclusion: We defined the clinicopathologic features of lung adenocarcinomas with high expression of PD-L1 and PD-L2. We further demonstrated the role of PD-L expression as a useful prognostic marker for lung adenocarcinoma.

Keywords: immunotherapy, lung cancer, prognostic markers, oncogenic driver mutations

\section{Introduction}

Lung cancer is the leading cause of cancer-related deaths worldwide. ${ }^{1}$ Despite significant advances in multidisciplinary cancer therapies, the overall prognosis for lung cancer patients remains poor. Novel treatment strategies, including immunotherapy, are under investigation to improve patients' prognosis.

Programmed death 1 (PD-1) is a coinhibitory receptor induced on activated $\mathrm{T}$ and B cells, ${ }^{2}$ and it plays a crucial role in tumor immune escape. ${ }^{3,4}$ Two ligands of PD-1, PD-L1 (B7-H1) and PD-L2 (B7-DC), have been identified as negative immune regulators by engaging PD-1 receptor. ${ }^{5}$ Several Phase I clinical trials demonstrated remarkable antitumor activity of both anti-PD-1 and anti-PD-L1 antibodies. ${ }^{6-8}$ An objective response rate of $10 \%-20 \%$ was observed in non-small-cell lung cancer patients. ${ }^{6,7}$ More interestingly, the responses were durable even in these heavily pretreated patients with 
lung cancer. ${ }^{6,7}$ Furthermore, immunohistochemical analysis of tumor specimens revealed a positive correlation between PD-L1 expression and objective responses. ${ }^{6,8}$

We carried out an immunohistochemical study of PD-L1 and PD-L2 expression in surgically resected lung adenocarcinoma and correlated their expression with clinicopathologic and molecular parameters, including adenocarcinoma histologic subtypes, patient prognosis, and common driver mutations.

\section{Materials and methods Patients and samples}

Lung adenocarcinoma samples were collected from patients who underwent surgical resection with curative intent in our institution from January 2008 to October 2009. Eligible patients were required to have sufficient tissue for immunohistochemical staining and comprehensive mutational analyses. Patients who received neoajuvant chemotherapy or had a history of malignant tumors were excluded.

Clinicopathologic variables collected for analyses included sex, age at diagnosis, smoking history, type of surgical resection, tumor histology, tumor differentiation, pathologic tumor-node-metastasis (TNM) stage in line with the seventh edition of the lung cancer staging system, ${ }^{9}$ adenocarcinoma subtypes according to the new International Association for the Study of Lung Cancer/American Thoracic Society/European Respiratory Society International Multidisciplinary Classification of Lung Adenocarcinoma, ${ }^{10}$ and postoperative adjuvant chemotherapy/radiotherapy. Disease recurrence and survival were observed in the follow-up clinic or obtained by telephone.

\section{Immunohistochemistry and interpretation}

Briefly, sections were deparaffinized and rehydrated, and were then treated with $3 \% \mathrm{H}_{2} \mathrm{O}_{2}$ to block endogenous peroxidase activity. Antigen retrieval was done by immersing slides in citrate buffer and microwaving. Nonspecific immunoglobulin binding was blocked using 10\% goat serum in phosphate-buffered saline (PBS) (Sigma-Aldrich, St. Louis, MO, USA). Sections were incubated with primary anti-PD-L1 antibody (SAB2900365; Sigma-Aldrich) at 1:300 or anti-PD-L2 antibody (HPA013411; SigmaAldrich) at 1:100. After incubation with the primary antibody, the slides were then washed by PBS and incubated with secondary antibodies followed by incubation with 3 , 3 '-diaminobenzidine (DAB). Sections were counterstained with hematoxylin.
The expression levels of PD-L1 and PD-L2 were measured by the semiquantitative quickscore method. ${ }^{11}$ Briefly, a quickscore for each sample was calculated by multiplying the general staining intensities throughout the whole section (0: negative; 1 : weak staining; 2 : intermediate staining; 3: strong staining) by the proportions of tumor cells staining positively throughout the section $(1: 0 \%-4 \% ; 2: 5 \%-19 \%$; 3: $20 \%-39 \%$; 4: 40\%-59\%; 5: 60\%-79\%; 6: 80\%-100\%), and ranged from $0-18 .{ }^{11}$ Two authors who were blinded to the clinical data assessed the immunostaining independently, and discrepancies in quickscores were resolved by reviewing the corresponding sections and by discussion.

\section{Mutational analysis}

Comprehensive mutational analyses of $E G F R, K R A S, A L K$, and $H E R 2$ were performed in lung adenocarcinomas as previously described. ${ }^{12-14}$ Briefly, ribonucleic acid was extracted as per standard protocol after frozen tissues were dissected into TRIzol $^{\circledR}$ (Life Technologies, Carlsbad, CA, USA), and was reverse transcribed into complementary deoxyribonucleic acid (cDNA). EGFR (exons 18-22), KRAS (exons 2-3), and HER2 (exons 18-21) were amplified by polymerase chain reaction (PCR) using cDNA. Primers were as follows: EGFR (forward: 5'-TGAAGGCTGTCCAACGAATG-3'; reverse: 5'-AGGCGTTCTCCTTTCTCCAG-3'), KRAS (forward: 5'-GAGAGGCCTGCTGAAAATGACTG-3'; reverse: 5'-TGGTGAATATCTTCAAATGATTTAGT-3'), and HER2 (forward: 5'-CCCTCTGACGTCCATCATCT-3'; reverse: 5'-GCAGGGTCTGGACAGAAGAA-3'). Amplified products were analyzed by direct dideoxynucleotide sequencing. A combined strategy of quantitative real-time PCR (qRT-PCR) and reverse transcriptase PCR (RT-PCR) were used to detect $A L K$ fusions, with validations using fluorescence in situ hybridization. ${ }^{13}$

\section{Statistical analysis}

Pearson's chi-squared test or Fisher's exact test was used to assess correlations between different immunoreactivity and clinicopathologic variables as well as mutational status. Kaplan-Meier method was used to draw the survival curves. Relapse-free and overall survival of patients with positive or negative immunostaining was compared using the log-rank test. Independent prognostic factors were identified through the Cox proportional hazards regression (forward likelihood ratio model). The predictive accuracy for overall survival was determined by the Harrell's concordance index (C-index) which ranges from 0.5 (no predictive power) to 1 (perfect prediction). ${ }^{15}$ The discriminatory ability of a prognostic 
model was measured by the Akaike information criterion (AIC) value (a smaller value indicates a better discriminatory ability). ${ }^{15}$ The statistical analyses were done using SPSS 16.0 (IBM Corporation, Armonk, NY, USA) and Stata 11.1 (StataCorp LP, College Station, TX, USA). All tests were two tailed. Statistical significance was set as $P<0.05$.

\section{Results}

A total of 143 lung adenocarcinoma samples were collected from 84 females and 59 males. The mean age of the patients was 58.6 years, ranging from 36-79 years. Detailed clinicopathologic and molecular characteristics are shown in Table 1. The expression of PD-L1 and PD-L2 was mainly located in the cell membrane and cytoplasm of tumor cells. Scattered expression of PD-L1 (weak to intermediate) and PD-L2 (weak) was also shown in macrophages. The median quickscores for PD-L1 and PD-L2 were 8 (range: 0-18) and 5 (range: 0-18), respectively, and were used as the cutoff values between positive and negative protein expression. Representative images of staining intensities of PD-Ls are shown in Figure 1.

\section{Correlation between PD-L expression and clinicopathologic and molecular features}

Positive PD-L1 expression was significantly associated with more advanced tumor (T) status, node involvement $(\mathrm{N})$ status, and pathologic stage. Histologically, lung adenocarcinomas with positive PD-L1 staining were less likely to be adenocarcinoma in situ or minimally invasive adenocarcinoma, and more likely to have solid predominant subtype. No significant correlation was observed between PD-L2 expression and clinicopathologic variables. PD-L expression was not correlated with any of the common driver mutations.

\section{Survival analysis}

In univariate analysis, PD-L1-positive patients had significantly poorer relapse-free survival (RFS) $(P<0.001)$ and overall survival (OS) $(P=0.002)$ than did PD-L1-negative patients (Figure 2). There was significant difference in OS ( $P=0.014)$ but only borderline significant difference in RFS $(P=0.071)$ between PD-L2 positive and PD-L2 negative patients. To correlate the combined PD-L expression with survival, we further divided the patients into three groups: (I) both PD-L1 and PD-L2 negative ( $\mathrm{n}=41$ ); (II) either PD-L1 or PD-L2 positive ( $\mathrm{n}=63$ ); and (III) both PD-L1 and PD-L2 positive ( $\mathrm{n}=39)$. Significant differences in RFS and OS were found between Group I and Group III $(P<0.001$ for RFS;
Table I Clinicopathologic and molecular characteristics according to PD-LI and PD-L2 expression

\begin{tabular}{|c|c|c|c|c|c|c|}
\hline \multirow[t]{2}{*}{ Variable } & \multicolumn{3}{|c|}{ PD-LI } & \multicolumn{3}{|c|}{ PD-L2 } \\
\hline & + & - & $P$ & + & - & $P$ \\
\hline Age (years) & & & 0.153 & & & 0.677 \\
\hline$\leq 58$ & 40 & 33 & & 35 & 38 & \\
\hline$>58$ & 30 & 40 & & 36 & 34 & \\
\hline Sex & & & 0.162 & & & 0.436 \\
\hline Female & 37 & 47 & & 44 & 40 & \\
\hline Male & 33 & 26 & & 27 & 32 & \\
\hline Smoking history & & & 0.728 & & & 0.127 \\
\hline Never smokers & 47 & 47 & & 51 & 43 & \\
\hline Ever smokers & 23 & 26 & & 20 & 29 & \\
\hline Differentiation & & & 0.376 & & & 0.332 \\
\hline Poor & 29 & 25 & & 24 & 30 & \\
\hline Moderate or well & 41 & 48 & & 47 & 42 & \\
\hline T status & & & 0.034 & & & 0.053 \\
\hline TI & 26 & 40 & & 27 & 39 & \\
\hline T2-T4 & 44 & 33 & & 44 & 33 & \\
\hline $\mathrm{N}$ status & & & 0.024 & & & 0.677 \\
\hline No & 29 & 44 & & 35 & 38 & \\
\hline NI/N2 & 41 & 29 & & 36 & 34 & \\
\hline Pathologic stage & & & 0.005 & & & 0.553 \\
\hline 1 & 24 & 42 & & 31 & 35 & \\
\hline II-III & 46 & 31 & & 40 & 37 & \\
\hline \multicolumn{7}{|l|}{ Histologic subtypes } \\
\hline AIS & 0 & 1 & 1.000 & 0 & I & 1.000 \\
\hline MIA & 0 & 6 & 0.028 & I & 5 & 0.209 \\
\hline Lepidic & 4 & 4 & 1.000 & 2 & 6 & 0.275 \\
\hline Papillary & 12 & 15 & 0.603 & 16 & II & 0.268 \\
\hline Acinar & 31 & 33 & 0.912 & 37 & 27 & 0.079 \\
\hline Solid & 21 & 11 & 0.032 & 14 & 18 & 0.449 \\
\hline Micropapillary & 0 & 1 & 1.000 & 0 & I & 1.000 \\
\hline IMA & I & 2 & 1.000 & I & 2 & 1.000 \\
\hline Enteric & I & 0 & 0.490 & 0 & I & 1.000 \\
\hline \multicolumn{7}{|l|}{ Mutational status } \\
\hline EGFR mutation & 37 & 39 & 0.946 & 36 & 40 & 0.561 \\
\hline KRAS mutation & 4 & 3 & 0.715 & 4 & 3 & 0.719 \\
\hline HER2 mutation & 2 & 5 & 0.442 & 4 & 3 & 0.719 \\
\hline$A L K$ fusion & 3 & 6 & 0.494 & 6 & 3 & 0.326 \\
\hline
\end{tabular}

Notes: The median quickscores for PD-LI (8) and PD-L2 (5) were used as the cutoff values between positive $(+)$ and negative $(-)$ protein expression. Bold indicates significant at $P<0.05$. Values in + and - columns represent $n$.

Abbreviations: AIS, adenocarcinoma in situ; IMA, invasive mucinous adenocarcinoma; MIA, minimally invasive adenocarcinoma; PD-L, programmed death I ligand.

$P<0.001$ for OS $)$ as well as Group II and Group III ( $P=0.002$ for RFS; $P=0.010$ for OS). RFS and OS of patients positive for either PD-L1 or PD-L2 tended to be worse than that of patients who were negative for both, although statistical significance was not achieved. T stage (T2-T4 versus T1; $P<0.001$ for RFS; $P=0.034$ for OS), N stage (N1/N2 versus N0; $P<0.001$ for RFS; $P<0.001$ for OS), and pathologic stage (stage II/III versus stage I; $P<0.001$ for RFS; $P<0.001$ for $\mathrm{OS}$ ) were also significantly associated with survival. 


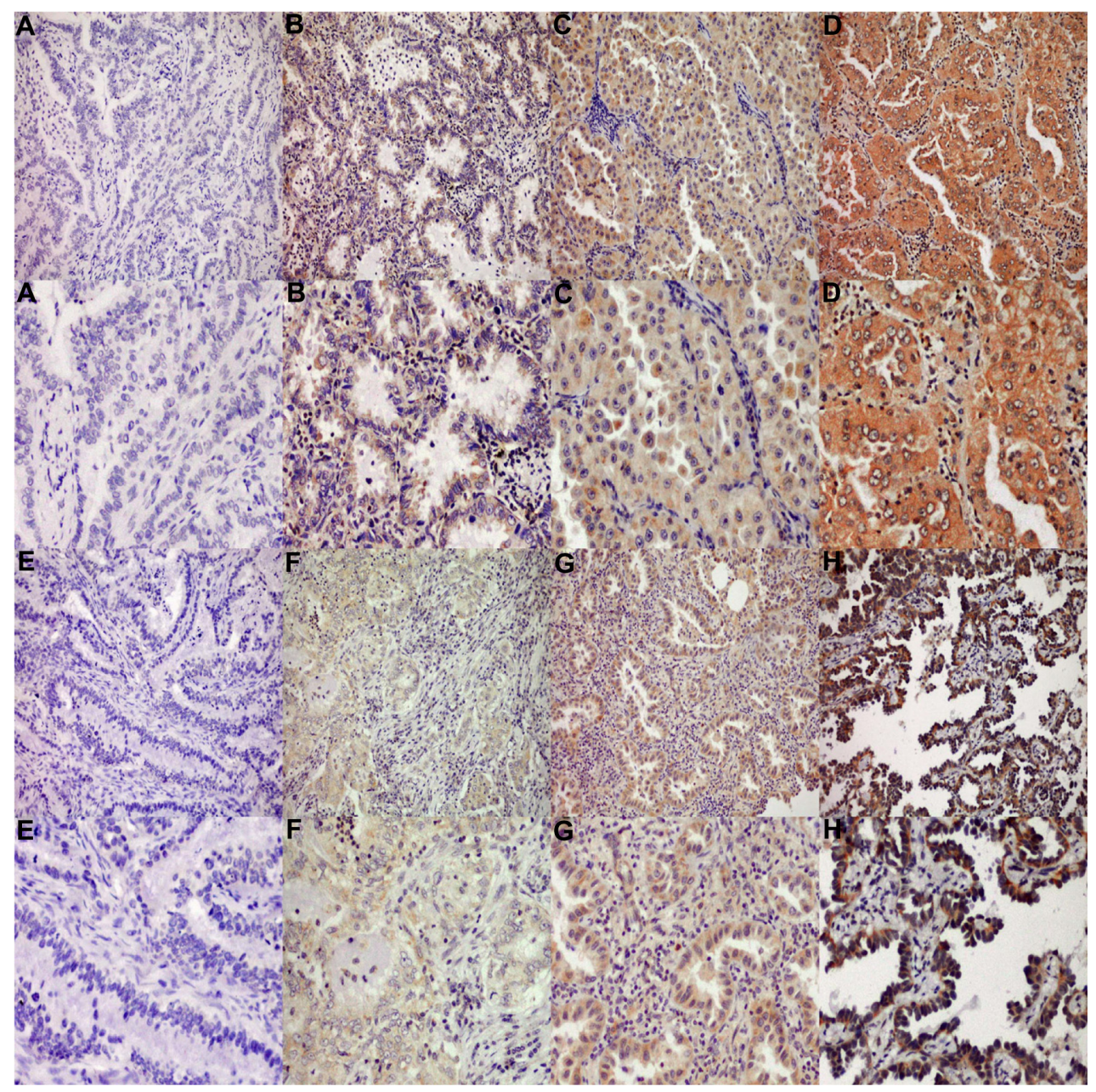

Figure I Representative images of staining intensities of PD-Ls.

Notes: Representative images of negative (A and E), weak (B and F), intermediate (C and $\mathbf{G})$, and strong (D and $\mathbf{H})$ staining intensities of PD-LI (A-D) and PD-L2 (E-H) in lung adenocarcinoma.

Abbreviation: PD-L, programmed death I ligand.

Mutational status of EGFR, KRAS, HER2, or ALK was not significantly associated with RFS or OS.

To determine the prognostic accuracy of PD-L expression, we used multivariate Cox regression model adjusted for age, sex, smoking history, type of surgical resection (lobectomy versus bi-lobectomy/pneumonectomy), differentiation, TNM stage, histologic subtypes, mutational status, and postoperative chemotherapy/radiotherapy. Both PD-L1 (odds ratio [OR] $=1.984,95 \%$ confidence interval $[\mathrm{CI}]=1.010-3.894 ; P=0.047)$ and PD-L2 expression $(\mathrm{OR}=2.328,95 \% \mathrm{CI}=1.201-4.512 ; P=0.012)$ were independent predictors of poor overall survival. We also assessed the combined prognostic value of PD-Ls expression (Group III versus Group I/II) in multivariate analysis, and found that both PD-L1 and PD-L2 positive expression status $(\mathrm{OR}=2.540,95 \% \mathrm{CI}=1.347-4.791 ; P=0.004)$ and pathologic stage (stage II/III versus stage I, OR $=4.971$,
$95 \%$ CI $=2.188-11.294$ ) were the only two independent predictors of poor overall survival.

We further calculated the C-index and AIC value to measure the prognostic accuracy of PD-L expression in conjunction with the current lung cancer staging system (Table 2). Pathologic stage alone had a C-index of 0.694 and an AIC value of 362.74. Higher C-index values ( 0.741 for PD-L1; 0.742 for PD-L2) and lower AIC values (359.54 for PD-L1; 358.71 for PD-L2) can be achieved by adding PD-L1 or PD-L2 expression to disease stage. When the combined PD-L expression and stage were used together to predict overall survival, the $\mathrm{C}$-index increased to 0.763 , and the AIC value decreased to 356.08 .

\section{Discussion}

Lung adenocarcinoma is a disease characterized by driver mutation-defined molecular subsets, each with distinct 

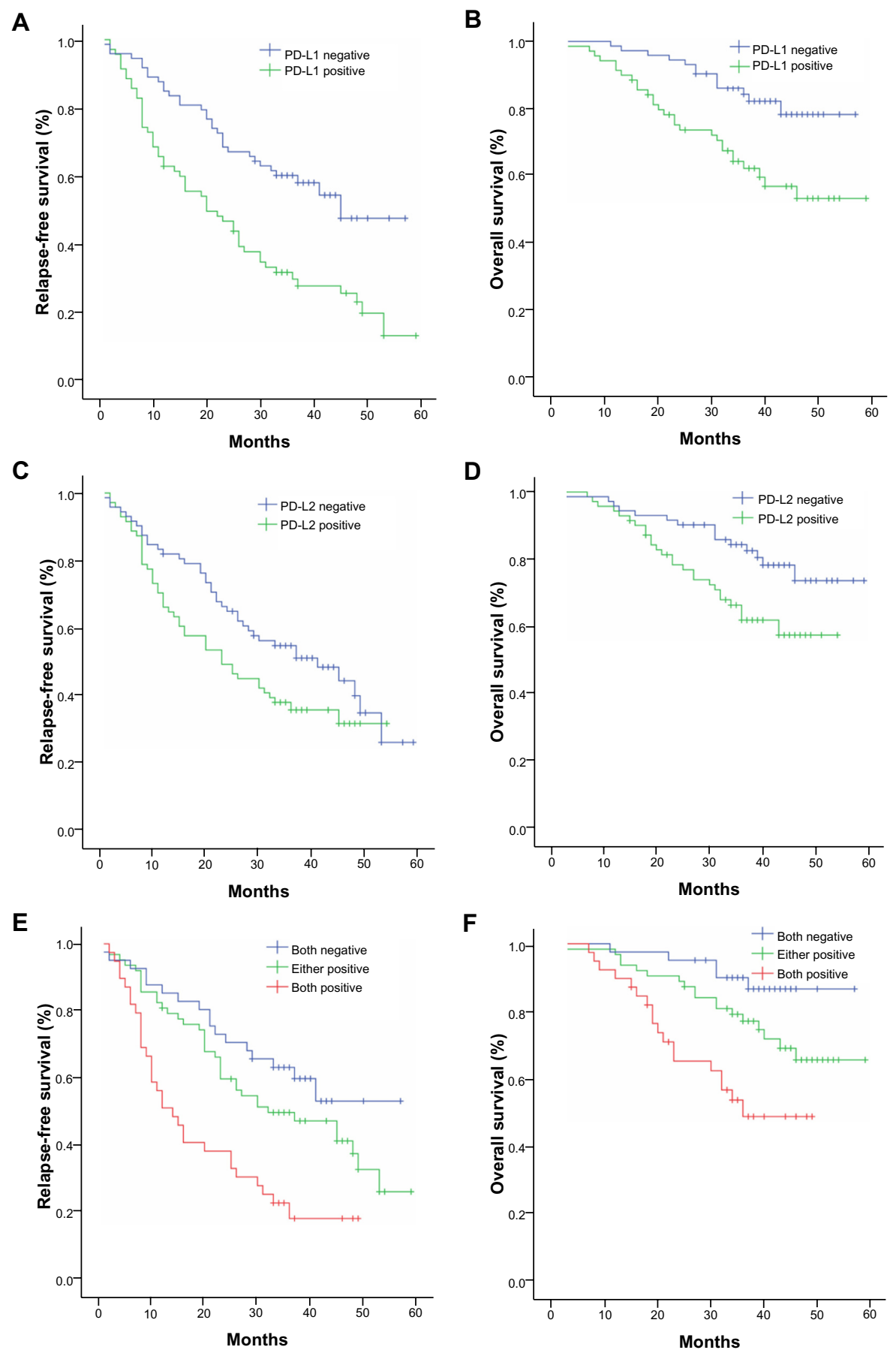

Figure 2 Relapse-free survival and overall survival in PD-LI and PD-L2 positive and negative patients.

Notes: (A) Relapse-free survival according to PD-LI expression $(P<0.00 I)$. (B) Overall survival according to PD-LI expression ( $P=0.002)$. (C) Relapse-free survival according to PD-L2 expression $(P=0.071)$. (D) Overall survival according to PD-L2 expression $(P=0.014)$. (E) Relapse-free survival according to PD-LI and PD-L2 expression $(P<0.00$ I for "both positive" versus "both negative"; $P=0.002$ for "both positive" versus "either positive"). (F) Overall survival according to PD-LI and PD-L2 expression $(P<0.00$ I for "both positive" versus "both negative"; $P=0.010$ for "both positive" versus "either positive").

Abbreviation: PD-L, programmed death I ligand.

clinicopathologic features and potentials for targeted therapies. Now, anti-PD-1/PD-L1 antibodies add to the weapons against this dreadful cancer type. Cancer cell-expressed PD-L1 was showed to induce apoptosis of antigen-specific T cells. ${ }^{16}$ Inhibition of the PD-1/PD-L interaction enhanced immune responses in vitro $^{17}$ and mediated preclinical antitumor activity. ${ }^{16,18}$ Besides the objective response rate of $10 \%-20 \%$ reported by the two clinical trials treating non-small-cell lung cancer patients with anti-PD-1/PD-L1 antibodies, the most intriguing fact is that the responses 
Table 2 Comparison of the prognostic accuracies of pathologic stage and PD-L expression

\begin{tabular}{lll}
\hline Model & C-index & AIC \\
\hline Stage & 0.694 & 362.74 \\
PD-LI expression + stage & 0.741 & 359.54 \\
PD-L2 expression + stage & 0.742 & 358.7 I \\
Combined PD-L expression + stage & 0.763 & 356.08 \\
\hline
\end{tabular}

Abbreviations: AIC, Akaike information criteria; C-index, Harrell's concordance index; PD-L, programmed death I ligand.

were durable even in those patients highly pretreated with conventional chemotherapy and tyrosine kinase inhibitors. ${ }^{6,7}$ Therefore, it is appealing to explore the clinicopathologic characteristics and molecular associations of lung adenocarcinomas expressing PD-L1 or PD-L2, which might be candidates for anti-PD-1/PD-L1 immunotherapy.

Two previous studies investigated the expression of PD-L1 (or in combination with PD-L2) in non-small-cell lung cancer. ${ }^{19,20}$ However, both studies had relatively small samples that were not limited to lung adenocarcinoma. To our knowledge, the current study is the first to assess the expression of PD-L1 and PD-L2 in lung adenocarcinoma together with a comprehensive panel of molecular and clinicopathologic variables, including common driver mutations and histologic subtypes of lung adenocarcinoma.

Although no significant correlations were found between PD-L expression and common driver mutations in lung adenocarcinoma, distinct histologic patterns were observed in lung adenocarcinomas with high expression of PD-L1. No adenocarcinoma in situ or minimally invasive adenocarcinoma showed high expression of PD-L1, whereas PD-L1 immunostaining was positively associated with solid predominant subtype. These data indicated that PD-L1 might be preferably highly expressed in more invasive/aggressive adenocarcinoma subtypes, ${ }^{21}$ offering implications for selecting candidates for immunotherapy.

Positive PD-L1 expression was found to be correlated with more advanced $\mathrm{T}$ status, $\mathrm{N}$ status, and pathologic stage, suggesting its role as a marker of disease progression. The most interesting finding was that both PD-L1 and PD-L2 were independent predictors of poor prognosis for lung adenocarcinoma patients, which is consistent with previous reports on malignant melanoma, ${ }^{22}$ urothelial cancer, ${ }^{23}$ ovarian cancer, ${ }^{24}$ hepatocellular carcinoma, ${ }^{25}$ esophageal cancer, ${ }^{26}$ and pancreatic cancer. ${ }^{27}$ However, Konishi and colleagues ${ }^{20}$ investigated 52 surgically resected specimens of non-smallcell lung cancer and found no significant association between PD-L1 or PD-L2 expression and patient survival. This discrepancy might be explained by the relatively small number of patients and the inclusion of histological types other than adenocarcinoma in their study.

Although TNM staging is a reliable prognostic factor, we showed that the addition of PD-L status, especially a combination of PD-L1 and PD-L2 status, markedly improved the prognostic accuracy in lung adenocarcinoma patients. This finding suggested that PD-L status might be used as a predictor for prognosis as well as a predictor for anti-PD-1/ PD-L1 antibodies treatment, which provided a strengthened rationale for immunotherapy for lung adenocarcinomas with high PD-L expression.

In conclusion, we defined the clinicopathologic features of lung adenocarcinomas with high expression of PD-L1 and PD-L2. We further demonstrated the role of PD-L expression as useful prognostic markers for lung adenocarcinoma. These data have implications for anti-PD-1/PD-L1 immunotherapy for lung adenocarcinoma patients.

\section{Disclosure}

The authors report no conflicts of interest in this work.

\section{References}

1. Jemal A, Bray F, Center MM, Ferlay J, Ward E, Forman D. Global cancer statistics. CA Cancer J Clin. 2011;61(2):69-90.

2. Agata Y, Kawasaki A, Nishimura H, et al. Expression of the PD-1 antigen on the surface of stimulated mouse $\mathrm{T}$ and $\mathrm{B}$ lymphocytes. Int Immunol. 1996;8(5):765-772.

3. Nishimura H, Honjo T. PD-1: an inhibitory immunoreceptor involved in peripheral tolerance. Trends Immunol. 2001;22(5):265-268.

4. Watanabe N, Gavrieli M, Sedy JR, et al. BTLA is a lymphocyte inhibitory receptor with similarities to CTLA-4 and PD-1. Nat Immunol. 2003;4(7):670-679.

5. Keir ME, Butte MJ, Freeman GJ, Sharpe AH. PD-1 and its ligands in tolerance and immunity. Annu Rev Immunol. 2008;26:677-704.

6. Topalian SL, Hodi FS, Brahmer JR, et al. Safety, activity, and immune correlates of anti-PD-1 antibody in cancer. $N$ Engl J Med. 2012;366(26):2443-2454.

7. Brahmer JR, Tykodi SS, Chow LQ, et al. Safety and activity of antiPD-L1 antibody in patients with advanced cancer. $N$ Engl $J$ Med. 2012;366(26):2455-2465.

8. Brahmer JR, Drake CG, Wollner I, et al. Phase I study of single-agent anti-programmed death-1 (MDX-1106) in refractory solid tumors: safety, clinical activity, pharmacodynamics, and immunologic correlates. J Clin Oncol. 2010;28(19):3167-3175.

9. Detterbeck FC, Boffa DJ, Tanoue LT. The new lung cancer staging system. Chest. 2009;136(1):260-271.

10. Travis WD, Brambilla E, Noguchi M, et al. International association for the study of lung cancer/american thoracic society/european respiratory society international multidisciplinary classification of lung adenocarcinoma. J Thorac Oncol. 2011;6(2):244-285.

11. Detre S, Saclani Jotti G, Dowsett M. A "quickscore" method for immunohistochemical semiquantitation: validation for oestrogen receptor in breast carcinomas. J Clin Pathol. 1995;48(9):876-878.

12. Zhang Y, Sun Y, Pan Y, et al. Frequency of driver mutations in lung adenocarcinoma from female never-smokers varies with histologic subtypes and age at diagnosis. Clin Cancer Res. 2012;18(7):1947-1953.

13. Wang R, Pan Y, Li C, et al. The use of quantitative real-time reverse transcriptase PCR for 5' and 3' portions of ALK transcripts to detect ALK rearrangements in lung cancers. Clin Cancer Res. 2012;18(17):4725-4732. 
14. Sun Y, Ren Y, Fang Z, et al. Lung adenocarcinoma from East Asian never-smokers is a disease largely defined by targetable oncogenic mutant kinases. J Clin Oncol. 2010;28(30):4616-4620.

15. Harrell FE Jr, Califf RM, Pryor DB, Lee KL, Rosati RA. Evaluating the yield of medical tests. JAMA. 1982;247(18):2543-2546.

16. Dong H, Strome SE, Salomao DR, et al. Tumor-associated B7-H1 promotes T-cell apoptosis: a potential mechanism of immune evasion. Nat Med. 2002;8(8):793-800.

17. Fife BT, Pauken KE, Eagar TN, et al. Interactions between PD-1 and PD-L1 promote tolerance by blocking the TCR-induced stop signal. Nat Immunol. 2009;10(11):1185-1192.

18. Iwai Y, Ishida M, Tanaka Y, Okazaki T, Honjo T, Minato N. Involvement of PD-L1 on tumor cells in the escape from host immune system and tumor immunotherapy by PD-L1 blockade. Proc Natl Acad Sci U S A. 2002;99(19):12293-12297.

19. Mu CY, Huang JA, Chen Y, Chen C, Zhang XG. High expression of PD-L1 in lung cancer may contribute to poor prognosis and tumor cells immune escape through suppressing tumor infiltrating dendritic cells maturation. Med Oncol. 2011;28(3):682-688.

20. Konishi J, Yamazaki K, Azuma M, Kinoshita I, Dosaka-Akita H, Nishimura M. B7-H1 expression on non-small cell lung cancer cells and its relationship with tumor-infiltrating lymphocytes and their PD-1 expression. Clin Cancer Res. 2004;10(15):5094-5100.

21. Yoshizawa A, Motoi N, Riely GJ, et al. Impact of proposed IASLC/ ATS/ERS classification of lung adenocarcinoma: prognostic subgroups and implications for further revision of staging based on analysis of 514 stage I cases. Mod Pathol. 2011;24(5):653-664.
22. Hino R, Kabashima K, Kato Y, et al. Tumor cell expression of programmed cell death-1 ligand 1 is a prognostic factor for malignant melanoma. Cancer. 2010;116(7):1757-1766.

23. Nakanishi J, Wada Y, Matsumoto K, Azuma M, Kikuchi K, Ueda S. Overexpression of B7-H1 (PD-L1) significantly associates with tumor grade and postoperative prognosis in human urothelial cancers. Cancer Immunol Immunother. 2007;56(8):1173-1182.

24. Hamanishi J, Mandai M, Iwasaki M, et al. Programmed cell death 1 ligand 1 and tumor-infiltrating CD8+ T lymphocytes are prognostic factors of human ovarian cancer. Proc Natl Acad Sci U S A. 2007;104(9): 3360-3365.

25. Gao Q, Wang XY, Qiu SJ, et al. Overexpression of PD-L1 significantly associates with tumor aggressiveness and postoperative recurrence in human hepatocellular carcinoma. Clin Cancer Res. 2009;15(3): 971-979.

26. Ohigashi Y, Sho M, Yamada Y, et al. Clinical significance of programmed death-1 ligand-1 and programmed death-1 ligand-2 expression in human esophageal cancer. Clin Cancer Res. 2005;11(8): 2947-2953.

27. Nomi T, Sho M, Akahori T, et al. Clinical significance and therapeutic potential of the programmed death-1 ligand/programmed death-1 pathway in human pancreatic cancer. Clin Cancer Res. 2007;13(7): 2151-2157.
OncoTargets and Therapy

\section{Publish your work in this journal}

OncoTargets and Therapy is an international, peer-reviewed, open access journal focusing on the pathological basis of all cancers, potential targets for therapy and treatment protocols employed to improve the management of cancer patients. The journal also focuses on the impact of management programs and new therapeutic agents and protocols on

\section{Dovepress}

patient perspectives such as quality of life, adherence and satisfaction The manuscript management system is completely online and includes a very quick and fair peer-review system, which is all easy to use. Visit http://www.dovepress.com/testimonials.php to read real quotes from published authors. 